\title{
Biomarkers for antitumor activity of bevacizumab in gastric cancer models
}

Yoriko Yamashita-Kashima, Kaori Fujimoto-Ouchi", Keigo Yorozu, Mitsue Kurasawa, Mieko Yanagisawa, Hideyuki Yasuno and Kazushige Mori

\begin{abstract}
Background: Bevacizumab is a humanized monoclonal antibody to human vascular endothelial cell growth factor (VEGF) and has been used for many types of cancers such as colorectal cancer, non-small cell lung cancer, breast cancer, and glioblastoma. Bevacizumab might be effective against gastric cancer, because VEGF has been reported to be involved in the development of gastric cancer as well as other cancers. On the other hand, there are no established biomarkers to predict the bevacizumab efficacy in spite of clinical needs. Therefore, we tried to identify the predictive markers for efficacy of bevacizumab in gastric cancer patients by using bevacizumab-sensitive and insensitive tumor models.
\end{abstract}

Methods: Nine human gastric and two colorectal cancer mouse xenografts were examined for their sensitivity to bevacizumab. We examined expression levels of angiogenic factors by ELISA, bioactivity of VEGF by phosphorylation of VEGFR2 in HUVEC after addition of tumor homogenate, tumor microvessel density by CD31immunostaining, and polymorphisms of the VEGF gene by HybriProbe ${ }^{\mathrm{TM}}$ assay.

Results: Of the 9 human gastric cancer xenograft models used, GXF97, MKN-45, MKN-28, 4-1ST, SC-08-JCK, and SC-09-JCK were bevacizumab-sensitive, whereas SCH, SC-10-JCK, and NCI-N87 were insensitive. The sensitivity of the gastric cancer model to bevacizumab was not related to histological type or HER2 status. All tumors with high levels of VEGF were bevacizumab-sensitive except for one, SC-10-JCK, which had high levels of VEGF. The reason for the refractoriness was non-bioactivity on the phosphorylation of VEGFR2 and micro-vessel formation of VEGF, but was not explained by the VEGF allele or VEGF165b. We also examined the expression levels of other angiogenic factors in the 11 gastrointestinal tumor tissues. In the refractory models including SC-10-JCK, tumor levels of another angiogenic factor, bFGF, were relatively high. The VEGF/bFGF ratio correlated more closely with sensitivity to bevacizumab than with the VEGF level.

Conclusions: VEGF levels and VEGF/bFGF ratios in tumors were related to bevacizumab sensitivity of the xenografts tested. Further clinical investigation into useful predictive markers for bevacizumab sensitivity is warranted.

\section{Background}

Vascular endothelial growth factor (VEGF), a diffusible glycoprotein produced by normal and neoplastic cells, is an important regulator of physiologic and pathologic angiogenesis. Increased VEGF levels in serum or tumor tissue have been reported to correlate with poor survival; therefore, efficacy of anti-VEGF therapy is expected in clinical application $[1,2]$.

\footnotetext{
* Correspondence: ohuchikor@chugai-pharm.co.jp

Product Research Department, Chugai Pharmaceutical Co., Ltd., 200, Kajiwara, Kamakura, Kanagawa, Japan 247-8530
}

(c) 2012 Yamashita-Kashima et al; licensee BioMed Central Ltd. This is an Open Access article distributed under the terms of the Creative Commons Attribution License (http://creativecommons.org/licenses/by/2.0), which permits unrestricted use, distribution, and reproduction in any medium, provided the original work is properly cited.
Bevacizumab is a humanized monoclonal antibody to human VEGF that inhibits VEGF-mediated angiogenesis in many types of tumors. In the US and EU, bevacizumab is used in combination with standard chemotherapies for patients with colorectal cancer, non-small cell lung cancer, breast cancer, and glioblastoma. Although bevacizumab improves progression-free survival (PFS) in these cancers, it is not effective for all patients. Predictive markers of bevacizumab efficacy have been assessed in many clinical trials [3], however, no validated biomarker is available to predict bevacizumab efficacy and identify the patients who could benefit from bevacizumab. Therefore, it is important 
to investigate the biomarker of bevacizumab efficacy from the phase of clinical development for other cancer types.

Gastric cancer is one of the most malignant cancers and second leading cause of cancer death in the world [4]. The incidence is reportedly highest in Asia, South America, and Southern Europe [5]. Increased levels of VEGF expression have been found in gastric cancers as well as in tumors of lung, breast, thyroid, gastrointestinal tract, kidney, bladder, ovary, cervix, and pancreas, angiosarcomas and glioblastomas [6,7]. A previous report suggests the possibility of VEGF as a prognostic factor of gastric cancer [8]. Therefore, bevacizumab may also be effective against gastric cancers [9]. In the present study, the relationship between the efficacy of bevacizumab, selected biomarkers in gastric cancers and various histological types of gastric cancer has been examined.

\section{Methods}

Test agents

Bevacizumab was provided by F. Hoffman-La Roche (Nutley, NJ, USA) as a liquid and diluted with saline. Human immunoglobulin G (HuIgG) was purchased from MP Biomedicals, Inc. (Aurora, OH, USA) and was reconstituted with water and diluted with saline.

\section{Animals}

Male 5-week-old BALB/c-nu/nu mice (CAnN.Cg-Foxn1 $<$ nu $>$ /CrlCrlj nu/nu) were obtained from Charles River Japan (Yokohama, Japan). All animals were allowed to acclimatize and recover from shipping-related stress for 1 week prior to the study. The health of the mice was monitored by daily observation. Chlorinated water and irradiated food were provided ad libitum, and the animals were kept in a controlled light-dark cycle (12 h-12 h). Animal procedures were approved by the Institutional Animal Care and Use Committee at Chugai Pharmaceutical Co., Ltd.

\section{Cell lines and culture conditions}

Nine human gastric cancer cell lines and two human colorectal cancer cell lines were used in the present study. MKN-45 and MKN-28 were purchased from ImmunoBiochemical Laboratories Co., Ltd. (Fujioka, Japan). NCI$\mathrm{N} 87, \mathrm{SCH}$, and HUVEC were obtained from the American Type Culture Collection (Rockville, MD, USA), the Japan Health Science Foundation (Osaka, Japan), and KURABO (Osaka, Japan), respectively. MKN-45 was maintained in TIL Media medium (Immuno-Biochemical Laboratories) supplemented with $10 \%(\mathrm{v} / \mathrm{v}) \mathrm{FBS}$, and MKN-28, NCIN87, and SCH were maintained in RPMI-1640 supplemented with $10 \%(\mathrm{v} / \mathrm{v}) \mathrm{FBS}$ at $37^{\circ} \mathrm{C}$ under $5 \% \mathrm{CO}_{2}$. HUVEC was maintained in HuMedia-EG2 (KURABO) at $37^{\circ} \mathrm{C}$ under $5 \% \mathrm{CO}_{2}$. Cell lines 4-1ST, SC-08-JCK, SC-09JCK, SC-10-JCK and COL-16-JCK were purchased from the Central Institute for Experimental Animals (Yokohama, Japan). GXF97 and CXF280 were kindly provided by Prof. H. H. Fiebig (University of Freiberg, Freiberg, Germany). 4-1ST, SC-08-JCK, SC-09-JCK, SC-10-JCK, GXF97 and CXF280 were maintained in BALB/c-nu/nu mice by subcutaneous (sc) inoculation of pieces of the tumor tissue.

\section{In vivo tumor growth inhibition studies}

Each mouse was inoculated sc into the right flank with either $5 \times 10^{6}$ cells/mouse of human gastric cancer cell line MKN-45, MKN-28, NCI-N87 or SCH, or an $8-\mathrm{mm}^{3}$ piece of 4-1ST, SC-08-JCK, SC-09-JCK, SC-10-JCK, GXF97 or CXF280 tumor tissue. Several weeks after tumor inoculation, the mice were randomly allocated to control and treatment groups. The administration of anticancer agents was started when tumor volumes reached approximately 0.2 to $0.3 \mathrm{~cm}^{3}$. To evaluate the antitumor activity of the test agents, tumor volume and body weight were measured twice a week. Tumor volumes $(\mathrm{V})$ were estimated from the equation $\mathrm{V}=\mathrm{ab} / 2$, where $\mathrm{a}$ and $b$ are tumor length and width, respectively. The percentage of tumor growth inhibition (TGI\%) was calculated as described previously [10].

\section{Treatment of animals}

Bevacizumab was administered intraperitoneally (ip) once a week for 3 weeks.

\section{Histological classification by hematoxylin-eosin staining}

Xenograft tumor tissues were collected, formalin-fixed, and paraffin-embedded. Slide specimens were prepared by sectioning the tissue and staining with hematoxylin-eosin stain. The histological classification was determined according to the Japanese Classification of Gastric Carcinoma (13th Edition, June 1999, Japanese Gastric Cancer Association).

\section{IHC, and gene amplification of HER2}

HER2 protein expression and HER2 gene amplification in tumors were examined by IHC using HercepTest ${ }^{\circledR}$ and FISH using Pathvysion ${ }^{\circledR}$ respectively as described previously [11].

\section{Measurement of microvessel density in tumor tissues} Microvessel density (MVD) in tumor tissue was evaluated immunohistochemically using a monoclonal anti-mouse CD31 antibody (rat anti-mouse CD31 monoclonal antibody, clone MEC 13.3; BD Biosciences, NJ, USA). Tumor samples were collected $24 \mathrm{~h}, 96 \mathrm{~h}$ or 21 days after bevacizumab administration. Immunohistochemical staining was performed as described previously [12]. MVD (\%) was calculated from the ratio of the CD31-positive staining area to the total observation area in the viable region. 
Three to six fields per section $\left(0.4856 \mathrm{~mm}^{2}\right.$ each $)$ were randomly analyzed, excluding necrotic areas. Positive staining areas were calculated using imaging analysis software (Win Roof; Mitani Corporation, Fukui, Japan).

\section{Quantification of human or mouse VEGF and other angiogenic proteins in tumor tissues and mouse serum}

Blood and tumor samples were taken when tumors had reached a volume of approximately 0.3 to $0.5 \mathrm{~cm}^{3}$. Blood serum was immediately retrieved and tumors were immediately frozen in liquid nitrogen and stored at $-80^{\circ}$ C. Tumor samples were homogenized in PBS containing $0.05 \%$ Tween 20 and centrifuged $\left(4^{\circ} \mathrm{C}, 10,000 \times \mathrm{g}\right.$, $20 \mathrm{~min}$.). The resultant supernatant was used for the assays. Human VEGF, placental growth factor (PlGF), interleukin-8 (IL-8) and basic fibroblast growth factor (bFGF) were quantified using Quantikine ${ }^{\circledR}$ ELISA kits (R\&D Systems, Minneapolis, MN, USA). Human VEGF165b was quantified using the DuoSet ${ }^{\circledR}$ ELISA Development System (R\&D Systems). Mouse VEGF was quantified using a Mouse VEGF Assay Kit (ImmunoBiochemical Laboratories). Total protein levels in the samples were quantified using a DC protein assay kit (BioRad Laboratories, Hercules, CA, USA).

\section{VEGF genotyping}

DNA was extracted from the cultured cells. VEGF polymorphisms $(-2578,-1154,-634$ and 936 on VEGF genomic DNA) were genotyped with two fluorescent hybridization probes using LightCycler ${ }^{\mathrm{TM}}$ software. Briefly, the analysis is based on PCR amplification of the region surrounding the polymorphism sites, followed by slowly melting off the polymorphism-covering hybridized probe while continuously detecting the fluorescence. Melting off the hybridized probe from its target sequence causes the fluorescent signal to disappear, and this allows a narrow and reproducible estimation of the meltingpoint temperature. Subtle differences in melting points between a completely matched hybrid duplex and a duplex with a single nucleotide mismatch are detectable using LightCycler.

\section{HUVEC pVEGFR2 assay}

Tumor samples were homogenized in HuMedia-EB2 (KURABO) and centrifuged $\left(4^{\circ} \mathrm{C}, 10,000 \times\right.$ g, $\left.20 \mathrm{~min}\right)$. The supernatant was retrieved and used as the assay medium. VEGF concentration in the samples was measured as described above. HUVEC was seeded at a density of $3 \times$ $10^{5}$ cells/well into 6-well plates in HuMedia-EG2 and incubated for 1 day at $37^{\circ} \mathrm{C}$ under $5 \% \mathrm{CO}_{2}$. After that, the medium was changed to a serum-starved medium (HuMedia-EB2 containing $0.5 \%$ FBS). After overnight incubation, the medium was changed to an assay medium which had been pre-treated with bevacizumab or human IgG at $37^{\circ} \mathrm{C}$ for $30 \mathrm{~min}$. After 5 -min incubation at $37^{\circ} \mathrm{C}$ under $5 \% \mathrm{CO}_{2}$, the plates were immediately placed on ice and the cells washed with PBS. The cells were then lysed with ice-cold lysis buffer \#9 (R\&D systems). Lysates were centrifuged at $14,000 \times \mathrm{g}$ for $20 \mathrm{~min}$ at $4^{\circ} \mathrm{C}$. The pVEGFR2 was detected using western blot method and DuoSet $\mathrm{IC}^{\circledR}$ ELISA Development System (R\&D Systems). For western blot, antibodies against VEGFR2 and pVEGFR2 (Tyr1175) (\#2479 and \#2478 respectively, Cell Signaling Tschnology, Beverly, MA, USA) were used as the first antibodies. The proteins were detected by horseradish peroxidase-conjugated secondary antibodies (Santa Cruz Biotechnology, Santa Cruz, CA, USA) and visualized using ECL plus (GE Healthcare Life Science, Buckinghamshire, UK).

\section{Statistical analysis}

The statistical analysis was carried out using a SAS preclinical package (SAS Institute, Inc., Tokyo, Japan).

\section{Results}

Sensitivity to bevacizumab in human gastric cancer xenograft models

We examined the antitumor activity of bevacizumab in the MKN-45 human gastric xenograft model. Bevacizumab showed significant antitumor activity against MKN-45 tumors at doses ranging from $1.25 \mathrm{mg} / \mathrm{kg}$ to $20 \mathrm{mg} / \mathrm{kg}$ (Figure 1A). On day 22 (21 days after starting treatment), tumor growth inhibition rates (TGI\%) were 62\%, 76\%, and $79 \%$ at doses of $1.25,5$, and $20 \mathrm{mg} / \mathrm{kg}$, respectively. The maximum effective dose was $5 \mathrm{mg} / \mathrm{kg}$. Then we investigated $5 \mathrm{mg} / \mathrm{kg}$ of bevacizumab antitumor activity against human gastric cancer xenografts of various degrees of differentiation as shown in Figure 1B. Bevacizumab showed significant antitumor activity in GXF97, MKN-28, 4-1ST, SC-08-JCK and SC-09-JCK, with TGI\% values on day 22 of $78 \%, 56 \%, 68 \%, 75 \%$, and $55 \%$, respectively. Meanwhile, bevacizumab did not show significant antitumor activity in NCI-N87, SCH and SC-10-JCK, with TGI\% values on day 22 of $21 \%, 3 \%$ and $11 \%$, respectively (Figure $1 C$ ). We also tested the antitumor activity of bevacizumab against two colorectal tumors, COL-16-JCK and CXF280, as the control because bevacizumab is approved for clinical use in colorectal cancers, and we found the TGI\% to be 59\% and $40 \%$, respectively (Figure 1D). The degree of antitumor activity of bevacizumab in the gastric cancer models was comparable to that in the colorectal cancer models. No weight loss (> 20\%) was observed for any of the doses tested in either model (data not shown).

\section{Histological types of tumor tissues}

The degree of differentiation among the tumors was examined by using hematoxylin-eosin staining (Figure 1B). 


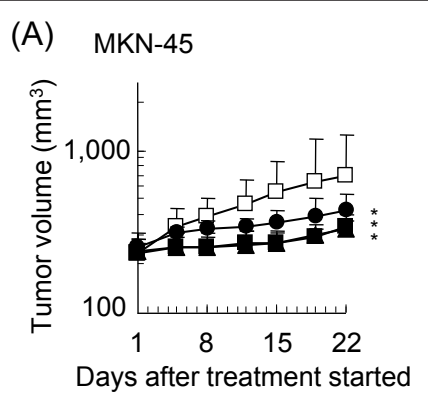

(B)

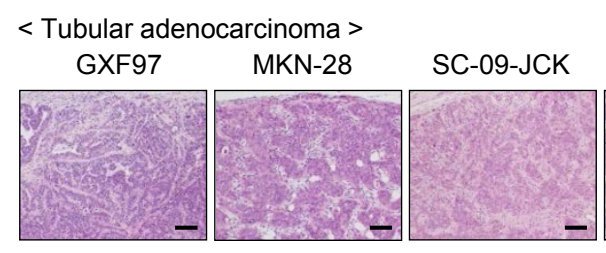

< Poorly differentiated adenocarcinoma > MKN-45

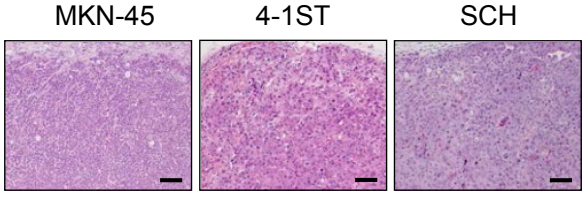

$\mathrm{SCH}$

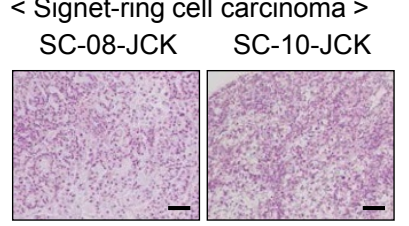

(C)

< Tubular adenocarcinoma >
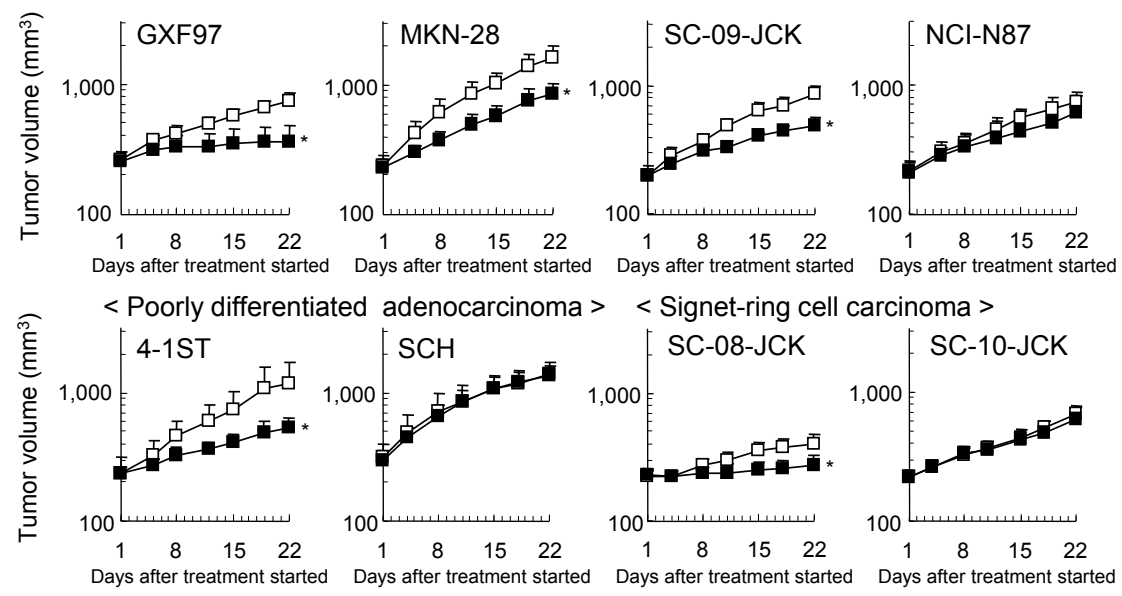

(D)

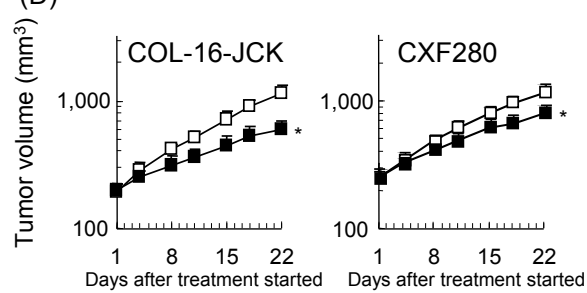

Figure 1 Antitumor activity of bevacizumab as a single agent in human gastric cancer xenograft models. (A) Mice bearing MKN-45 tumors were randomly divided into groups and HulgG or bevacizumab $(1.25,5,20 \mathrm{mg} / \mathrm{kg})$ was administered intraperitoneally, once a week for 3 weeks. $\square$ : human $\lg G 20 \mathrm{mg} / \mathrm{kg}$, $\bullet$ : bevacizumab1.25 mg/kg, : bevacizumab 5 mg/kg, $\mathbf{\Delta}$ : bevacizumab $20 \mathrm{mg} / \mathrm{kg}$. (B) Differentiation degree of each tumor was determined by hematoxylin-eosin staining. (C) Antitumor activity of bevacizumab $(5 \mathrm{mg} / \mathrm{kg})$ was evaluated in various types of gastric cancer xenograft models: GXF97, MKN-28, NCI-N87, 4-1ST, SCH, SC-08-JCK, SC-09-JCK, SC-10-JCK. $\square$ : human lgG 20 mg/kg, - bevacizumab $5 \mathrm{mg} / \mathrm{kg}$. (D) Antitumor activity of bevacizumab (5 mg/kg) was evaluated in two colorectal cancer xenograft models, COL-16-JCK and CXF280. 口: human lgG $20 \mathrm{mg} / \mathrm{kg}$, : bevacizumab $5 \mathrm{mg} / \mathrm{kg}$. Data points are mean + SD of tumor volume $\left(\mathrm{mm}^{3}\right)$. Statistically significant differences were shown as *: $P<0.05$ vs. control group by Wilcoxon test. ( $n=5-7 /$ group). 
GXF97, MKN-28, SC-09-JCK, and NCI-N87 were categorized as tubular adenocarcinoma, known as a well differentiated type. MKN-45, 4-1ST and SCH were categorized as poorly-differentiated adenocarcinoma and all of them were solid-type. SC-08-JCK and SC-10-JCK were categorized as signet-ring cell carcinoma. There was no correlation between the efficacy of bevacizumab and the degree of differentiation of the tumors, and bevacizumab showed antitumor activity against every differentiation type of tumor we tested as shown in Figure 1.

\section{HER2 status of tumor tissues}

HER2 status of tumor tissues which we examined bevasizumab sensitivity was shown in Table 1 . There was also no relevancy between the efficacy of bevacizumab and the HER2 status, as shown by the observation that the HER2-positive tumor 4-1ST was sensitive to bevacizumab but HER2-positive tumors NCI-N87, and SCH were insensitive to bevacizumab.

\section{Expression of human VEGF}

There is a possibility that the level of expression of human VEGF in tumor tissue is involved in the efficacy of bevacizumab, because bevacizumab is an anti-human VEGF monoclonal antibody. We quantified the expression level of human VEGF and VEGF165b in several gastric and colorectal tumor tissues (Figure 2A). We found that human VEGF was hardly expressed in bevacizumab-insensitive tumors $\mathrm{SCH}$ and NCI-N87 but, in the insensitive tumor SC-10-JCK, human VEGF was expressed. VEGF165b is reported to be an anti-angiogenic factor [13] and thus, could inhibit bevacizumab activity. However, it was not expressed to any extent in the tumors we investigated. Therefore, the effect of VEGF165b was considered to be negligible in the examined tumor models. Angiogenic human VEGF was more significantly expressed in bevacizumab-sensitive tumors compared with bevacizumab-

Table 1 HER2 expression in gastrointestinal cancer cell lines

\begin{tabular}{lcc}
\hline & \multicolumn{2}{c}{ HER2 status } \\
\cline { 2 - 3 } Cell line & IHC & FISH \\
\hline NCI-N87 & $2+$ & 8.4 \\
$4-1 S T$ & $3+$ & 5.3 \\
SCH & $2+$ & 2.0 \\
SC-09-JCK & 0 & 1.2 \\
SC-10-JCK & 0 & 1.2 \\
MKN-45 & 0 & 1.1 \\
MKN-28 & 0 & 1.0 \\
SC-08-JCK & 0 & 1.0 \\
GXF97 & 0 & 0.9 \\
\hline
\end{tabular}

HER2 protein expression and HER2 gene amplification in tumors were examined by IHC using HercepTest ${ }^{\mathbb{R}}$ and FISH using Pathvysion ${ }^{\mathbb{B}}$ respectively

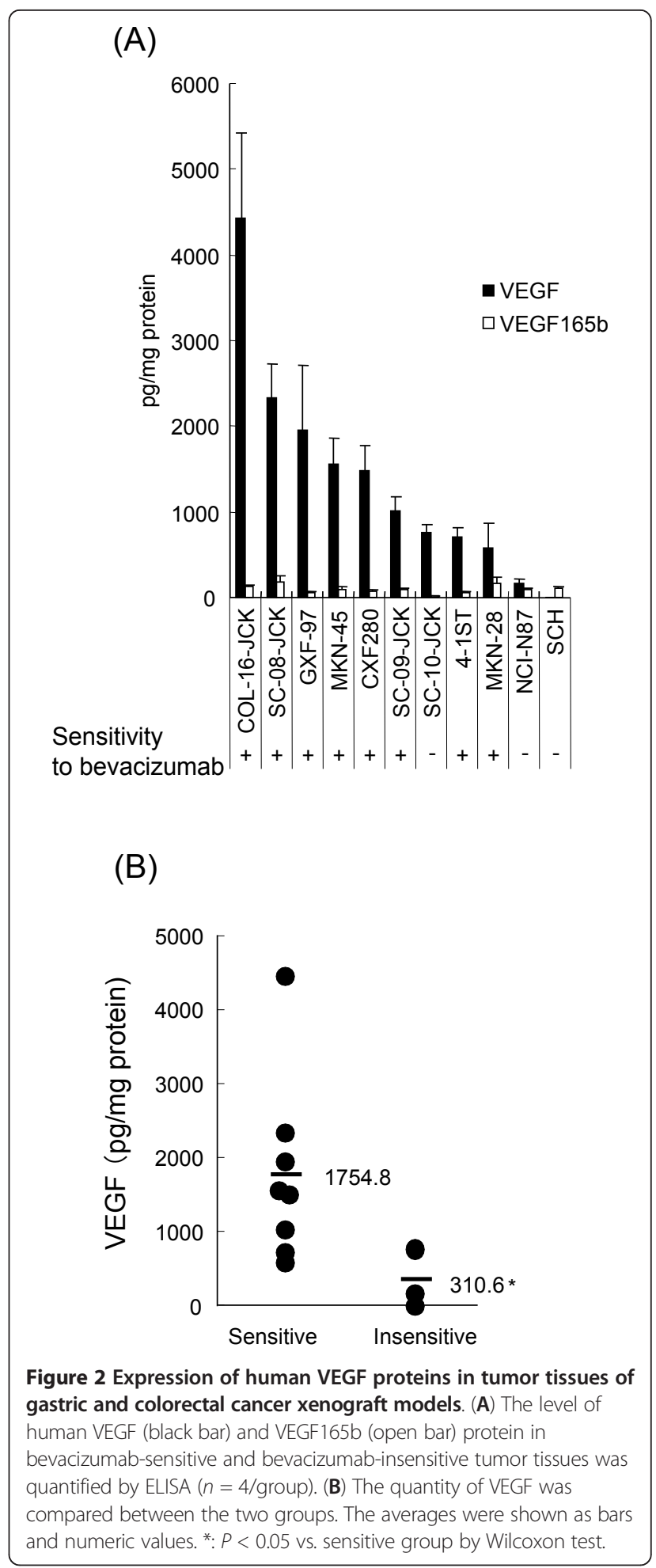

insensitive tumors (Figure 2B, $\mathrm{p}=0.0485$ ). The correlation coefficient $\left(\mathrm{r}^{2}\right)$ between TGI\% and the log concentration of human VEGF per mg protein in tumor tissue was 0.5312 . We also quantified the expression level of mouse VEGF in the tumors, but the correlation coefficient 
between TGI\% and mouse tumor VEGF was not observed $\left(r^{2}=0.0098\right)$. We also quantified human and mouse VEGF in serum, but the level of expression was below the limit of detection $(<31.25 \mathrm{pg} / \mathrm{mL})$.

\section{Polymorphisms of VEGF}

In clinical breast cancer patients, the efficacy of bevacizumab is reported to correlate to the status of VEGF polymorphism, the $-2578 \mathrm{~A}$ allele. Hence, we tried to examine the correlation of VEGF polymorphisms and efficacy, but statistical analysis could not be done because the A/A allele was only observed in the MKN-45 tumor. The level of VEGF in MKN-45 was not high, even though the -2578 allele exists in the VEGF promoter (Table 2).

\section{Bioactivity of VEGF in SC-10-JCK}

We examined whether the VEGF expressed in SC-10JCK was bioactive. Tumor tissue lysates of two bevacizumab-sensitive models, GXF97 (high VEGF expression) and 4-1ST (VEGF level equivalent to SC-10-JCK), and one bevacizumab-insensitive model, SC-10-JCK, with or without bevacizumab, were added to HUVEC, and the phosphorylation level of VEGFR2 in HUVEC was tested by western blot (Figure 3A). VEGF levels of the lysates of GXF97, 4-1ST and SC-10-JCK were 24.7, 8.0 and 4.7 $\mathrm{ng} / \mathrm{mL}$ respectively. VEGF derived from bevacizumabsensitive tumors (GXF97 and 4-1ST) phosphorylated VEGFR2. The VEGF diluted to $4.7 \mathrm{ng} / \mathrm{mL}$ (a concentration equal to SC-10-JCK-derived VEGF) still phosphorylated VEGFR2. All phosphorylation of VEGFR2 by VEGF from bevacizumab-sensitive tumors was reduced by bevacizumab. On the other hand, SC-10-JCK-derived VEGF did not phosphorylate VEGFR2 both in western blot and ELISA (Figure 3B). These results suggest that the VEGF in SC-10-JCK was not bioactive.

Table 2 VEGF polymorphisms in gastrointestinal cancer cell lines

\begin{tabular}{lcccc}
\hline & \multicolumn{4}{c}{ Genotype } \\
\cline { 2 - 5 } Cell line & $\mathbf{4} 578$ & $-\mathbf{1 1 5 4}$ & $\mathbf{- 6 3 4}$ & $\mathbf{9 3 6}$ \\
\hline MKN-45 & $\mathrm{A} / \mathrm{A}$ & $\mathrm{A} / \mathrm{A}$ & $\mathrm{G} / \mathrm{G}$ & $\mathrm{T} / \mathrm{T}$ \\
4-1ST & $\mathrm{C} / \mathrm{A}$ & $\mathrm{G} / \mathrm{A}$ & $\mathrm{G} / \mathrm{G}$ & $\mathrm{C} / \mathrm{C}$ \\
GXF97 & $\mathrm{C} / \mathrm{A}$ & $\mathrm{G} / \mathrm{A}$ & $\mathrm{C} / \mathrm{G}$ & $\mathrm{C} / \mathrm{C}$ \\
MKN-28 & $\mathrm{C} / \mathrm{C}$ & $\mathrm{G} / \mathrm{A}$ & $\mathrm{C} / \mathrm{C}$ & $\mathrm{C} / \mathrm{C}$ \\
SC-09-JCK & $\mathrm{C} / \mathrm{C}$ & $\mathrm{G} / \mathrm{A}$ & $\mathrm{G} / \mathrm{G}$ & $\mathrm{C} / \mathrm{C}$ \\
SC-08-JCK & $\mathrm{C} / \mathrm{C}$ & $\mathrm{G} / \mathrm{A}$ & $\mathrm{C} / \mathrm{C}$ & $\mathrm{T} / \mathrm{T}$ \\
NCI-N87 & $\mathrm{C} / \mathrm{A}$ & $\mathrm{G} / \mathrm{A}$ & $\mathrm{G} / \mathrm{G}$ & $\mathrm{C} / \mathrm{C}$ \\
SC-10-JCK & $\mathrm{C} / \mathrm{A}$ & $\mathrm{G} / \mathrm{A}$ & $\mathrm{G} / \mathrm{G}$ & $\mathrm{C} / \mathrm{T}$ \\
SCH & $\mathrm{C} / \mathrm{C}$ & $\mathrm{G} / \mathrm{A}$ & $\mathrm{C} / \mathrm{G}$ & $\mathrm{C} / \mathrm{C}$ \\
\hline
\end{tabular}

VEGF polymorphisms (-2578, $-1154,-634$ and 936 on VEGF genomic DNA) were examined by using LightCycler ${ }^{\mathrm{TM}}$

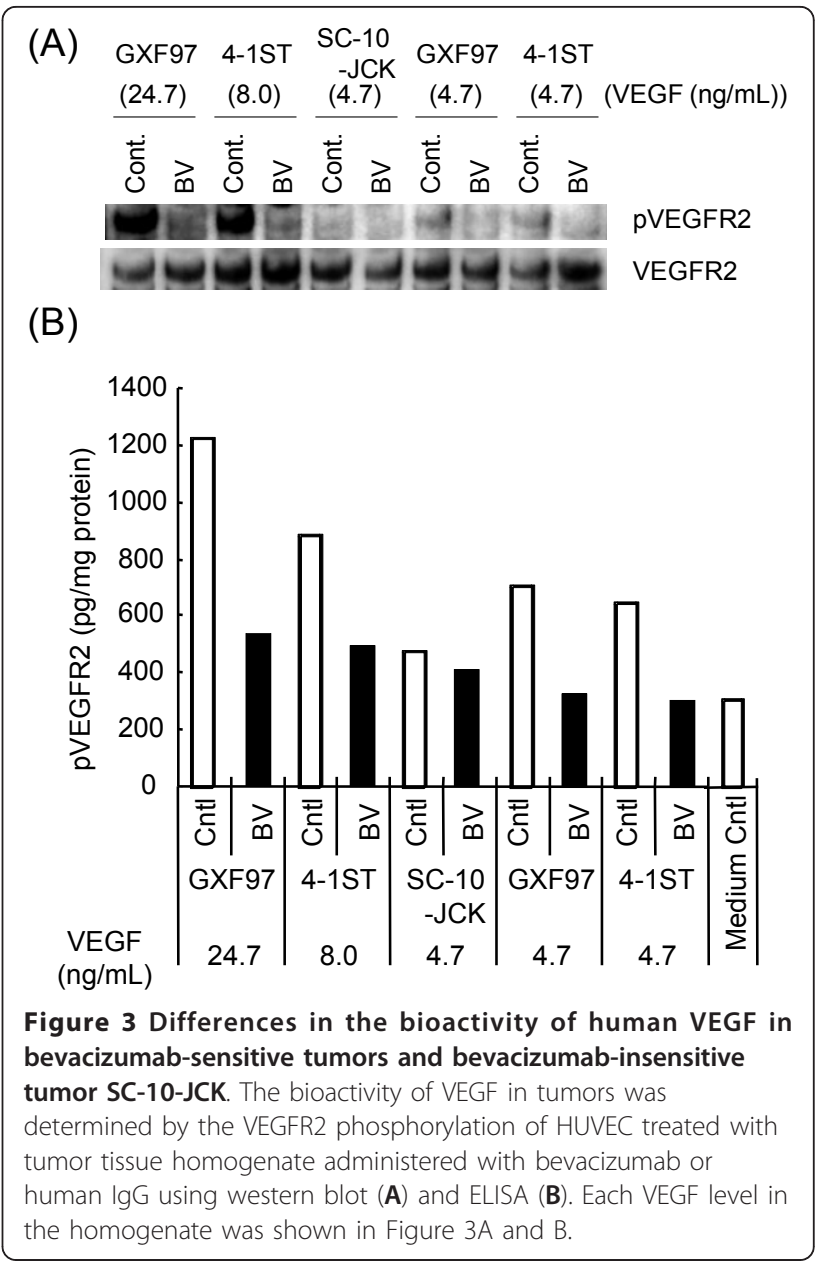

Anti-angiogenic activity of bevacizumab

We also examined changes in MVD in bevacizumab-sensitive and -insensitive tumors after bevacizumab treatment using CD31-staining (Figure 4A). In the bevacizumabsensitive tumor models, GXF97 and SC-08-JCK, a significant decrease of MVD was observed at $96 \mathrm{~h}$ after singletreatment of bevacizumab and at 21 days after the 3-week treatment (Figure $4 \mathrm{~B}$ and $4 \mathrm{C}$ ). On the other hand, MVD had not changed at any time point after bevacizumab treatment in the bevacizumab-insensitive tumor models, SC-10-JCK and SCH (Figure 4D and 4E). The decrease of MVD was corresponded to the antitumor activity of bevacizumab, but was detected earlier than the decrease of tumor volume (Figure 4F-I). We also examined the change in MVD in the GXF97 tumor samples at the lower dose (1 $\mathrm{mg} / \mathrm{kg}$ ), at which bevacizumab had not shown efficacy on day 22 (Figure 4J). In correlation with the efficacy of bevacizumab, MVD had not changed in GXF97 tumor tissue $96 \mathrm{~h}$ after bevacizumab treatment at the low dose. Therefore we considered that bevacizumab showed antitumor activity by inhibiting tumor angiogenesis. 


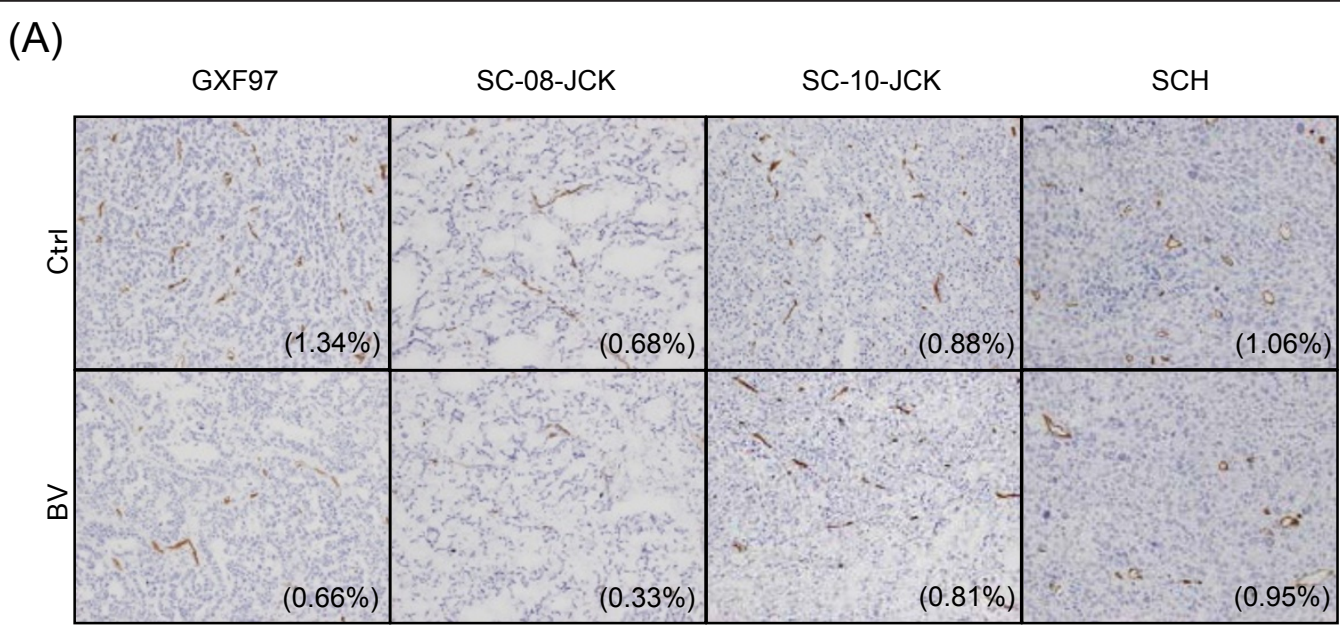

MVD at $96 \mathrm{~h}$ after BV treatment.

( ) is the ratio of CD31-immunostaining positive area to total observation area

(B)

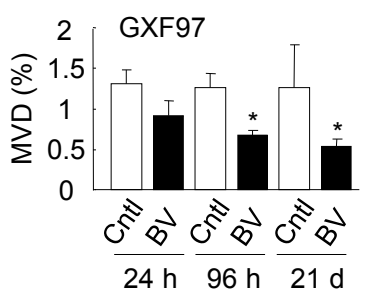

$(\mathrm{F})$

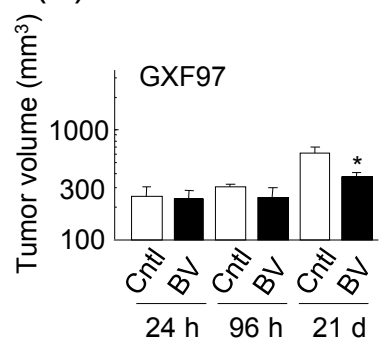

(C)

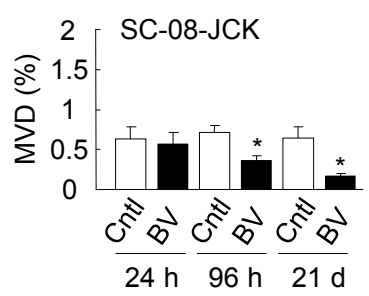

(G)

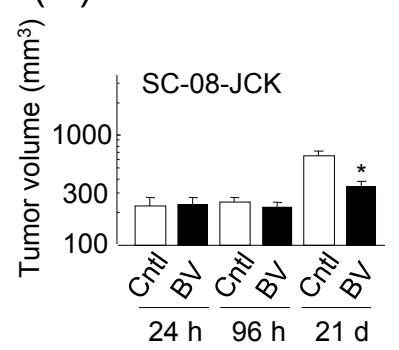

(D)

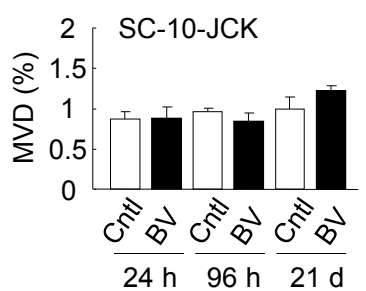

$(\mathrm{H})$

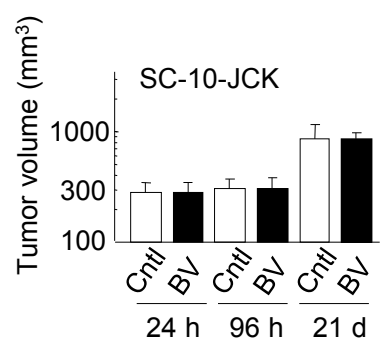

$(\mathrm{E})$

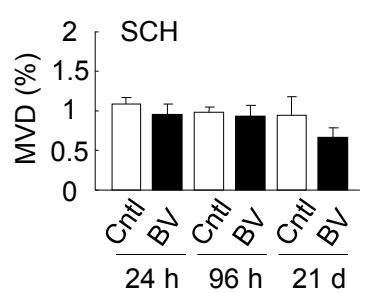

(I)

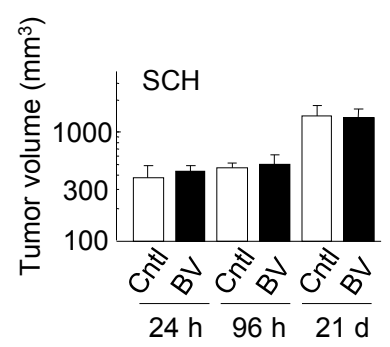

(J) GXF97 BV $1 \mathrm{mg} / \mathrm{kg}$

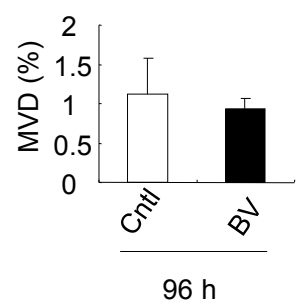

Figure 4 Change in MVD after bevacizumab treatment. (A) CD31 immunostaining in tumor tissue at $96 \mathrm{~h}$ after treated with HulgG (Ctrl) and bevacizumab (BV) in GXF97, SC-08-JCK, SC-10-JCK and SCH models. (B-E) The MVD in tumor tissue was determined by calculating the ratio of CD31-immunostaining positive area to the total observation area at $24 \mathrm{~h}, 96 \mathrm{~h}$ and 21 days $(21 \mathrm{~d})$ after bevacizumab treatment $(n=3-6 / g r o u p)$. (F-I) Tumor volumes were measured on the day of MVD evaluation. (J) MVD after treatment of bevacizumab at 1 mg/kg was evaluated in GXF97 $(n=4)$. *: $P<0.05$ vs. control group by Wilcoxon test. 
Other angiogenic factors as the bevacizumab resistance factor in tumor tissues

The MVD results after bevacizumab treatment suggested that angiogenic factors other than VEGF are involved in angiogenesis in bevacizumab-insensitive tumors. Therefore, we investigated the expression levels of angiogenic factors bFGF, IL- 8 and PlGF in tumor tissues, all of which are reported to be angiogenic factors for tumors (Table 3). Each one as a single factor did not significantly correlate with bevacizumab efficacy (Figure 5A). However, the VEGF/bFGF ratio in bevacizumab-insensitive tumors was significantly lower than that in bevacizumabsensitive tumors $(1.30 \%$ vs. $12.4 \%$, Figure $5 \mathrm{~B}, \mathrm{p}=0.0242)$.

\section{Discussion}

Gastric cancer has long been classified according to degree of differentiation and histopathologic type. Recently, HER2 expression has been included as a classification method in response to the positive results of a phase III study showing that addition of trastuzumab to a combination of capecitabine (or 5FU) and CDDP in patients with HER2-overexpressing gastric cancers prolonged overall survival [14]. In light of this, we attempted to clarify the types of gastric cancers for which bevacizumab would be effective. We used human cancer xenograft models developed by inoculating human gastric cancer cells into T-cell-deficient mice to investigate the efficacy of bevacizumab. Human VEGF secreted from human cancer cells is known to have an effect on mouse endothelial cells and results in angiogenesis in xenograft tumor tissues. In MKN-45, bevacizumab showed significant antitumor activity, although the dose dependency was not strong. A low dose of bevacizumab $(1.25 \mathrm{mg} / \mathrm{kg})$ was sufficient to produce antitumor activity. We examined the antitumor activity of bevacizumab in 9 human gastric cancer xenograft models of various degrees of differentiation, tumor types, and HER2 expression.
However, the sensitivity of the gastric cancer models to bevacizumab was found to be unrelated to the histological type or the HER2 status of the tumors. The antitumor activity of bevacizumab in the gastric cancer xenograft models was similar to that observed in colorectal cancer [15], for which bevacizumab has shown survival and clinical benefit.

Next, we examined other biomarker candidates of bevacizumab efficacy using sensitive and insensitive gastric tumor models. Because VEGF is the molecular target of bevacizumab, the tumor level of VEGF is thought to influence the sensitivity to bevacizumab. Tumor VEGF levels were significantly lower in the xenograft models insensitive to bevacizumab than in the sensitive models; however, mouse tumor VEGF showed no differences between sensitive and insensitive tumors. Human and mouse blood VEGF were lower than the detection limit in these models, and thus they were of little concern. In the SC-10-JCK model, in spite of VEGF expression, bevacizumab did not exhibit efficacy. To clarify the role of VEGF as a biomarker candidate of bevacizumab sensitivity, we investigated subtypes of VEGF in more detail. Various isoforms of VEGF are known such as the reportedly anti-angiogenic VEGF, VEGF165b [13]. VEGF165b binds to bevacizumab [16]. In our study, VEGF165b exhibited $100 \%$ cross-reactivity to VEGF ELISA (data not shown) so we thought that VEGF165b expression would be high in SC-10-JCK. However, the level of VEGF165b was low in the SC-10-JCK model. Additionally, VEGF165b expression was low in all of the tumor tissues we examined. In the gastrointestinal models tested, VEGF165b is not a concern. Because bevacizumab binds to all angiogenic isoforms of VEGF, such as VEGF121, VEGF165, and VEGF189 [17], identification of these isoforms is not necessary. In clinical breast cancer treatment, polymorphisms of VEGF have been found to correlate with the efficacy of the bevacizumab and paclitaxel combination [18]. The survival time of patients with

Table 3 Expression of angiogenic factors in gastrointestinal cancer cell lines

\begin{tabular}{llcccc}
\hline Cell line & Sensitivity to bevacizumab & VEGF & PIGF & IL-8 & bFGF \\
\hline COL-16-JCK & + & $4430 \pm 991$ & $<15.6$ & $360 \pm 171$ & $268 \pm 75.3$ \\
SC-08-JCK & + & $2330 \pm 402$ & $<15.6$ & $<31.3$ & $145 \pm 25.9$ \\
GXF97 & + & $1950 \pm 753$ & $<15.6$ & $362 \pm 134$ & $107 \pm 63.6$ \\
MKN-45 & + & $1560 \pm 310$ & $<15.6$ & $65.9 \pm 9.38$ & $183 \pm 43.0$ \\
CXF280 & + & $1480 \pm 290$ & $<15.6$ & $<31.3$ & $77.6 \pm 16.5$ \\
SC-09-JCK & + & $1020 \pm 163$ & $<15.6$ & $165 \pm 60.3$ & $151 \pm 13.3$ \\
4-1ST & + & $706 \pm 106$ & $<15.6$ & $<31.3$ & $87.7 \pm 5.47$ \\
MKN-28 & + & $577 \pm 285$ & $16.4 \pm 8.22$ & $<31.3$ & $200 \pm 27.8$ \\
SC-10-JCK & - & $760 \pm 80.2$ & $<15.6$ & $198 \pm 96.5$ & $231 \pm 105$ \\
NCl-N87 & - & $171 \pm 52.5$ & $<15.6$ & $34.4 \pm 10.8$ & $1090 \pm 328$ \\
SCH & - & $<31.3$ & $1210 \pm 205$ & $<31.3$ & $38.4 \pm 18.6$ \\
\hline
\end{tabular}

Mean \pm SD (pg/mg protein)

The expression of human VEGF, PIGF, IL-8 and bFGF in tumor tissues were examined by ELISA 


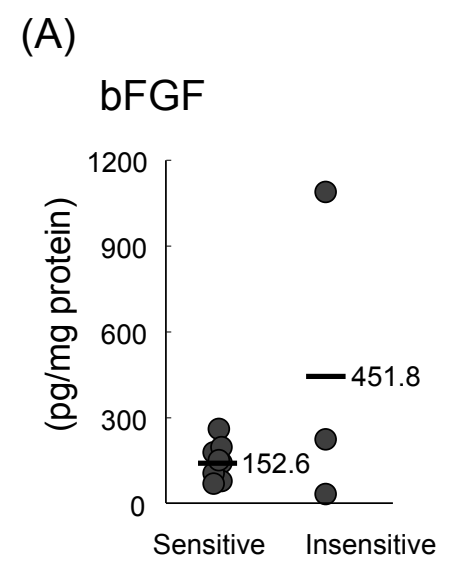

(B)

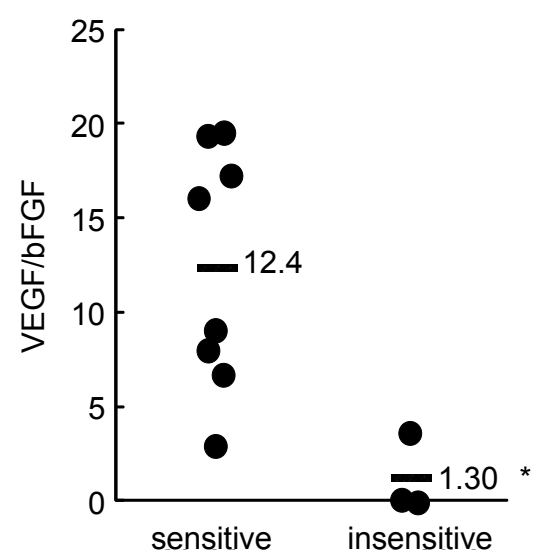

Figure 5 Expression of human bFGF protein in tumor tissues of gastric and colorectal cancer xenograft models. (A) The level of human bFGF, PIGF, and IL-8 proteins in tumor tissues sensitive (9 models) or insensitive (3 models) to bevacizumab was quantified by ELISA ( $n=4$ ). Open circle means less than the detection limit of ELISAs. The averages were shown as bars and numeric values. (B) The ratio of VEGF to bFGF was compared between the two groups. * $P<0.05$ vs. sensitive group by Wilcoxon test.

the 2578 AA allele or 1154 A allele of VEGF was longer than that of others. However, VEGF 2578 and 1154 of SC10-JCK were CA and GA, respectively, and the type of allele was the same as with the sensitive tumors 4-1ST and GXF97. The VEGF allele did not distinguish sensitivity to bevacizumab in these models. Next, to clarify whether the VEGF of SC-10-JCK had biological activity, the effect of a tumor homogenate of SC-10-JCK on the induction of VEGFR phosphorylation on HUVEC was examined. The tumor homogenate of SC-10-JCK did not have phosphorylation activity in contrast to that of 4-1ST or GXF97. Furthermore, the MVD in the SC-10-JCK xenograft model treated with bevacizumab was not reduced, indicating that the VEGF of SC-10-JCK did not show angiogenic activity. These results suggest the possibility of existence of nonactive VEGF in tumors.

We attempted to clarify both the biomarker for and the mechanisms of bevacizumab. The inhibition of MVD in tumor tissue is reported to be a major mechanism of bevacizumab efficacy, and so we compared MVD inhibition with efficacy in bevacizumab-sensitive and -insensitive tumor models. The existence of MVD inhibition corresponded with antitumor efficacy. In the present study, the MVD inhibition prior to antitumor efficacy was shown, and MVD inhibition was found to be a mechanism of bevacizumab efficacy. Our results indicated that other angiogenic factors except for VEGF are involved in biomarkers for bevacizumab resistance. We examined tumor bFGF, IL- 8 and PlGF, all of which are reported to be angiogenic factors for tumors [19-21]. When we compared the ratio of bFGF to VEGF with bevacizumab efficacy to clarify the extent of the role of VEGF in angiogenesis, the ratio of VEGF to bFGF in bevacizumab-insensitive tumors was clearly lower than that in bevacizumab-sensitive tumors. It is thought that an intimate cross-talk of bFGF and VEGF family is exist during angiogenesis and bFGF 
increases neuroplin-1, a co-receptor for VEGF, expression in human vascular smooth muscle cell that in turn enhance the effect of VEGF on cell migration and promote neovascularizaion [22]. In the present study, we did not examine the bioactivity of bFGF in the bevacizumab insensitive tumors. It is necessary to investigate the effect of bFGF on VEGF-dependent angiogenesis and on its inhibition by bevacizumab in the future. Neither VEGF/PlGF nor VEGF/IL-8 showed significant results (data not shown). PlGF has been reported to form a homodimer or heterodimer with VEGF and bind the resulting VEGFR in angiogenesis [23]. However, the cell lines we examined did not express PlGF, except for $\mathrm{SCH}$, so we examined the relationship between $\mathrm{PlGF}$ and bevacizumab in $\mathrm{SCH}$ in vitro. HUVEC growth induction with a PlGF/VEGF heterodimer was inhibited by bevacizumab (data not shown), suggesting that the number of heterodimers of PlGF and VEGF would also be a biomarker for bevacizumab sensitivity. Further examination is needed. PlGF homodimers did not show HUVEC growth induction, and we did not examine the effect of bevacizumab on HUVEC growth.

Some reports have indicated that bevacizumab exhibits direct cell growth inhibition against tumor cells [24,25]. However, in the gastric cancer cell lines we examined, no direct antitumor activity was observed in spite of the growth inhibition shown by bevacizumab in the xenograft model of the same cancer cell line (data not shown).

\section{Conclusions}

In the present study of gastric cancer treatment, we found that levels of VEGF and the VEGF/bFGF ratio have a close correlation with sensitivity to bevacizumab in the tested cell lines. There was no correlation between sensitivity to bevacizumab and the histological classification according to the degree of differentiation or HER2 status of the tumor cells. Clinical evidence is expected.

\section{Acknowledgements}

We thank Ms. Sally Matsuura (Research Planning \& Coordination Department, Chugai) for helpful advices about drafting the manuscript.

\section{Authors' contributions}

YYK designed experiments, performed experiments and statistical analysis, interpreted results and drafted manuscript. KFO established the study concept and conducted critical revision to manuscript. KY, MK, MY and $\mathrm{HY}$ performed experiments. KM performed peer reviewing the final draft. All authors have read and approved the final manuscript.

\section{Competing interests}

The authors declare that they have no competing interests.

Received: 24 August 2011 Accepted: 25 January 2012 Published: 25 January 2012

\section{References}

1. Kuroi $\mathrm{K}$, Toi M: Circulating angiogenesis regulators in cancer patients. Int J Biol Markers 2001, 16(1):5-26.
2. Poon RT, Fan ST, Wong J: Clinical implications of circulating angiogenic factors in cancer patients. J Clin Oncol 2001, 19(4):1207-1225.

3. Jubb AM, Harris AL: Biomarkers to predict the clinical efficacy of bevacizumab in cancer. Lancet Oncol 2010, 11(12):1172-1183.

4. Jemal A, Bray F, Center MM, Ferlay J, Ward E, Forman D: Global cancer statistics. CA Cancer J Clin 2011, 61(2):69-90.

5. Kamangar F, Dores GM, Anderson WF: Patterns of cancer incidence, mortality, and prevalence across five continents: defining priorities to reduce cancer disparities in different geographic regions of the world. J Clin Oncol 2006, 24(14):2137-2150.

6. Ferrara N, Davis-Smyth T: The biology of vascular endothelial growth factor. Endocr Rev 1997, 18(1):4-25.

7. von Marschall Z, Cramer T, Hocker M, Burde R, Plath T, Schirner M, Heidenreich R, Breier G, Riecken EO, Wiedenmann B, Rosewicz S: De novo expression of vascular endothelial growth factor in human pancreatic cancer: evidence for an autocrine mitogenic loop. Gastroenterology 2000, 119(5):1358-1372.

8. Kido S, Kitadai Y, Hattori N, Haruma K, Kido T, Ohta M, Tanaka S, Yoshihara M, Sumii K, Ohmoto Y, Chayama K: Interleukin 8 and vascular endothelial growth factor -prognostic factors in human gastric carcinomas? Eur J Cancer 2001, 37(12):1482-1487.

9. Maeda K, Kang SM, Onoda N, Ogawa M, Sawada T, Nakata B, Kato Y, Chung YS, Sowa M: Expression of p53 and vascular endothelial growth factor associated with tumor angiogenesis and prognosis in gastric cancer. Oncology 1998, 55(6):594-599.

10. Fujimoto-Ouchi K, Sekiguchi F, Tanaka Y: Antitumor activity of combinations of anti-HER-2 antibody trastuzumab and oral fluoropyrimidines capecitabine/5'-dFUrd in human breast cancer models. Cancer Chemother Pharmacol 2002, 49(3):211-216.

11. Fujimoto-Ouchi K, Sekiguchi F, Yasuno H, Moriya Y, Mori K, Tanaka Y: Antitumor activity of trastuzumab in combination with chemotherapy in human gastric cancer xenograft models. Cancer Chemother Pharmacol 2007, 59(6):795-805.

12. Yanagisawa M, Yorozu K, Kurasawa M, Nakano K, Furugaki K, Yamashita $Y$, Mori K, Fujimoto-Ouchi K: Bevacizumab improves the delivery and efficacy of paclitaxel. Anticancer Drugs 2010, 21(7):687-694

13. Woolard J, Wang WY, Bevan HS, Qiu Y, Morbidelli L, Pritchard-Jones RO, Cui TG, Sugiono M, Waine E, Perrin R, Foster R, Digby-Bell J, Shields JD, Whittles CE, Mushens RE, Gillatt DA, Ziche M, Harper SJ, Bates DO VEGF165b, an inhibitory vascular endothelial growth factor splice variant: mechanism of action, in vivo effect on angiogenesis and endogenous protein expression. Cancer Res 2004, 64(21):7822-7835.

14. Bang YJ, Van Cutsem E, Feyereislova A, Chung HC, Shen L, Sawaki A, Lordick F, Ohtsu A, Omuro Y, Satoh T, Aprile G, Kulikov E, Hill J, Lehle M, Ruschoff J, Kang YK: Trastuzumab in combination with chemotherapy versus chemotherapy alone for treatment of HER2-positive advanced gastric or gastro-oesophageal junction cancer (ToGA): a phase 3, openlabel, randomised controlled trial. Lancet 2010, 376(9742):687-697.

15. Yanagisawa M, Fujimoto-Ouchi K, Yorozu K, Yamashita Y, Mori K: Antitumor activity of bevacizumab in combination with capecitabine and oxaliplatin in human colorectal cancer xenograft models. Oncol Rep 2009, 22(2):241-247.

16. Varey AH, Rennel ES, Qiu Y, Bevan HS, Perrin RM, Raffy S, Dixon AR, Paraskeva C, Zaccheo O, Hassan AB, Harper SJ, Bates DO: VEGF 165 b, an antiangiogenic VEGF-A isoform, binds and inhibits bevacizumab treatment in experimental colorectal carcinoma: balance of pro- and antiangiogenic VEGF-A isoforms has implications for therapy. $\mathrm{Br} J$ Cancer 2008, 98(8):1366-1379

17. Kim KJ, Li B, Houck K, Winer J, Ferrara N: The vascular endothelial growth factor proteins: identification of biologically relevant regions by neutralizing monoclonal antibodies. Growth Factors 1992, 7(1):53-64.

18. Schneider BP, Wang M, Radovich M, Sledge GW, Badve S, Thor A, Flockhart DA, Hancock B, Davidson N, Gralow J, Dickler M, Perez EA Cobleigh M, Shenkier T, Edgerton S, Miller KD: Association of vascular endothelial growth factor and vascular endothelial growth factor receptor-2 genetic polymorphisms with outcome in a trial of paclitaxel compared with paclitaxel plus bevacizumab in advanced breast cancer: ECOG 2100. J Clin Oncol 2008, 26(28):4672-4678.

19. Presta M, Dell'Era P, Mitola S, Moroni E, Ronca R, Rusnati M: Fibroblast growth factor/fibroblast growth factor receptor system in angiogenesis. Cytokine Growth Factor Rev 2005, 16(2):159-178. 
20. Waugh DJ, Wilson C: The interleukin-8 pathway in cancer. Clin Cancer Res 2008, 14(21):6735-6741.

21. De Falco S, Gigante B, Persico MG: Structure and function of placental growth factor. Trends Cardiovasc Med 2002, 12(6):241-246.

22. Korc M, Friesel RE: The role of fibroblast growth factors in tumor growth. Curr Cancer Drug Targets 2009, 9(5):639-651.

23. Autiero M, Waltenberger J, Communi D, Kranz A, Moons L, Lambrechts $D$, Kroll J, Plaisance S, De Mol M, Bono F, Kliche S, Fellbrich G, Ballmer-Hofer K, Maglione D, Mayr-Beyrle U, Dewerchin M, Dombrowski S, Stanimirovic D, Van Hummelen P, Dehio C, Hicklin DJ, Persico G, Herbert JM, Shibuya M, Collen D, Conway EM, Carmeliet P: Role of PIGF in the intra- and intermolecular cross talk between the VEGF receptors Flt1 and Flk1. Nat Med 2003, 9(7):936-943

24. Liang Y, Hyder SM: Proliferation of endothelial and tumor epithelial cells by progestin-induced vascular endothelial growth factor from human breast cancer cells: paracrine and autocrine effects. Endocrinology 2005, 146(8):3632-3641.

25. Kim SJ, Seo JH, Lee YJ, Yoon JH, Choi CW, Kim BS, Shin SW, Kim YH, Kim JS: Autocrine vascular endothelial growth factor/vascular endothelial growth factor receptor-2 growth pathway represents a cyclooxygenase2-independent target for the cyclooxygenase-2 inhibitor NS-398 in colon cancer cells. Oncology 2005, 68(2-3):204-211.

\section{Pre-publication history}

The pre-publication history for this paper can be accessed here: http://www.biomedcentral.com/1471-2407/12/37/prepub

doi:10.1186/1471-2407-12-37

Cite this article as: Yamashita-Kashima et al:: Biomarkers for antitumor activity of bevacizumab in gastric cancer models. BMC Cancer 2012

$12: 37$.

\section{Submit your next manuscript to BioMed Central} and take full advantage of:

- Convenient online submission

- Thorough peer review

- No space constraints or color figure charges

- Immediate publication on acceptance

- Inclusion in PubMed, CAS, Scopus and Google Scholar

- Research which is freely available for redistribution

Submit your manuscript at www.biomedcentral.com/submit 